\title{
Hypereosinophilic syndrome with cardiac involvement in a patient with multiple malignancies
}

\author{
Mihaela Ioana Dregoesc ${ }^{1,2}$, Adrian Corneliu Iancu ${ }^{1,2}$, Alexandra Alina Lazãr ${ }^{2}$, \\ Şerban Bãlãnescu ${ }^{3}$
}

${ }^{1}$ Cardiology Department, "Iuliu Haţieganu" University of Medicine and Pharmacy, Cluj-Napoca, ${ }^{2 " N i c u l a e ~ S t a ̃ n c i o i u " ~}$ Heart Institute, Cluj-Napoca, 3 "Elias" University Hospital, "Carol Davila” University of Medicine and Pharmacy, Bucharest, Romania

\section{To the Editor,}

A 72-year-old woman with a history of multiple malignancies was referred to the Cardiology Department in April 2017 for dyspnea on exertion. She had a history of bilateral mastectomy in 1986 for breast cancer and total thyroidectomy in 1993 for papillary carcinoma. In 2015 she underwent a corporeal-caudal pancreatectomy for an intra-ductal papillary mucinous neoplasm. Bone and liver metastases were documented three months prior to the current admission.

On transthoracic echocardiography, an echogenic mass covered the apex and the lateral wall of the left ventricle and markedly reduced its cavity. Left ventricular systolic function could not be accurately quantified due to the presence of the intraventricular mass. Moderate mitral regurgitation, a dilated left atrium and a mild pericardial effusion were also identified (fig 1 A,B).

Given past medical history, left ventricular metastases were suspected. However, marked peripheral blood eosinophilia $(3740 / \mu \mathrm{l})$, accounting for $53.6 \%$ of the circulating leukocytes, raised awareness of Loeffler endocarditis. Cardiac magnetic resonance imaging revealed a non-dilated left ventricle, with an ejection fraction of $50 \%$. Areas of subendocardial hypoperfusion adjoined regions covered by a massive cardiac thrombus, with a heterogeneous structure, more recent in the periphery

Received 05.05.2018 Accepted 11.06.2018

Med Ultrason

2018, Vol. 20, No 3, 399-400, DOI: 10.11152/mu-1574

Corresponding author: Prof. Adrian C. Iancu

19-21 Calea Moților, Cluj-Napoca,

Romania, 400001

Phone: +40744751027 , Fax: +40264595090

E-mail: adrian_iancu@hotmail.com

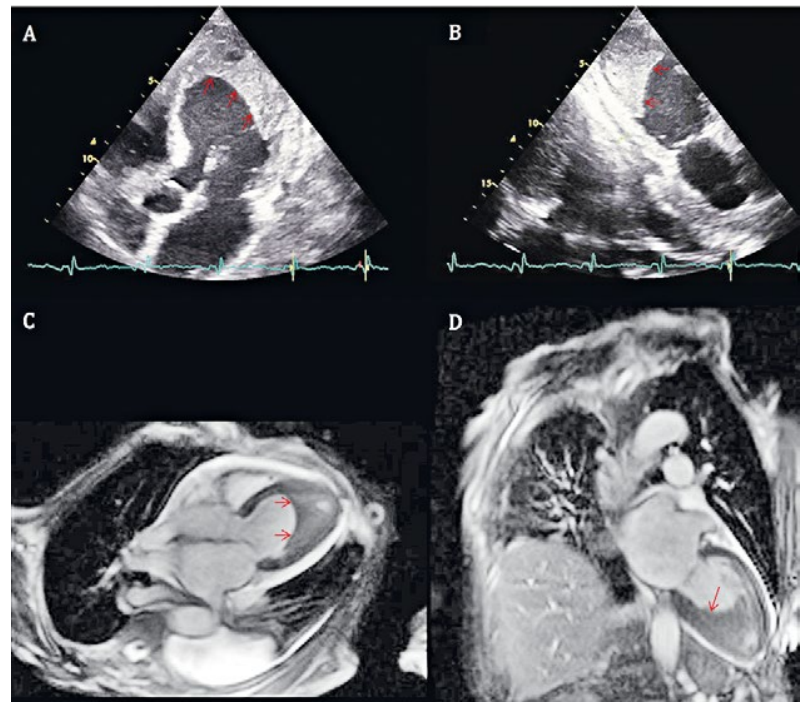

Fig 1. A - Echocardiography apical five chamber view: an echogenic mass covers the apex and the lateral wall of the left ventricle; B - Echocardiography apical two chamber view: the left ventricular cavity is markedly reduced by an echogenic mass that covers the apex; $\mathrm{C}$ - Cardiac magnetic resonance imaging transverse view: massive left ventricular thrombus; D - Cardiac magnetic resonance imaging frontal view: the cardiac thrombus has a heterogeneous structure, more recent in the periphery and older in its central regions.

and older in its central regions (fig 1 C,D). Subendocardial hypoperfusion and thrombus formation represent classic findings in the second stage of eosinophilic endocarditis [1].

Retrospectively, an increased eosinophil count had been constantly noticed in her blood tests since 2015 . Parasitic infections and allergies were ruled out. The patient was started on anticoagulants and heart failure 
medication but she declined cytoreductive therapy. The course of the disease was unfavorable. The patient died two months later from sudden cardiac sudden cardiac death.

Paraneoplastic eosinophilia is usually mild, but absolute counts may occasionally exceed $1500 / \mu 1$. These patients may remain asymptomatic or develop signs of endorgan dysfunction many years after the eosinophilia is first noted [2]. The most common mechanisms accountable for hypereosinophilia in patients with malignancies regard the production of cytokines by the primary tumor [3], the eosinophilotactic response due to tumor necrosis or the increased production of eosinophils as a consequence of bone marrow invasion [4].

\section{References}

1. Debl K, Djavidani B, Buchner S, et al. Time course of eosinophilic myocarditis visualized by CMR. J Cardiovasc Magn Reson 2008;2013:21.

2. Chen YY, Khoury P, Ware JM, et al. Marked and persistent eosinophilia in the absence of clinical manifestations. J Allergy Clin Immunol 2014;133:1195.

3. Anagnostopoulos GK, Sakorafas GH, Kostopoulos P, et al. Disseminated colon cancer with severe peripheral blood eosinophilia and elevated serum levels of interleukine-2, interleukine-3, interleukine-5, and GM-CSF. J Surg Oncol 2005;89:273-275.

4. Rothenberg ME. Eosinophilia. N Engl J Med 1998;338:1592-1600.

\title{
A rare sonographic finding for suprascapular nerve entrapment: engorged suprascapular artery not vein
}

\author{
Wei-Ting Wu ${ }^{1}$, Ke-Vin Chang ${ }^{1}$, Levent Özçakar ${ }^{2}$, Carlo Martinoli ${ }^{3}$
}

${ }^{1}$ Department of Physical Medicine and Rehabilitation and Community and Geriatric Research Center, National Taiwan University Hospital, Bei-Hu Branch and National Taiwan University College of Medicine, Taipei, Taiwan, ${ }^{2}$ Department of Physical and Rehabilitation Medicine, Hacettepe University Medical School, Ankara, Turkey, ${ }^{3}$ Department of Health Sciences (DISSAL), University of Genoa, Genoa, Italy

\section{To the Editor}

A 56-year-old woman has been complaining of chronic left shoulder pain for the last one year, after having been forcefully pushed on her left shoulder. She described the pain as intermittent and shooting, and also aggravating during active shoulder movements. On the first visit, Hawkin's, painful arc and lift off tests were positive, but the shoulder radiography revealed no aspect suggesting fracture or dislocation. The initial ultrasound (US) exami-

Received 09.05.2018 Accepted 10.06.2018

Med Ultrason

2018, Vol. 20, No 3, 400-401, DOI: 10.11152/mu-1579

Corresponding author: Ke-Vin Chang, $\mathrm{MD}, \mathrm{PhD}$

Department of Physical Medicine and

Rehabilitation, National Taiwan University

Hospital, Bei-Hu Branch, No. 87 Neijiang St,

Wanhua District, Taipei City 108, Taiwan

E-mail: kvchang011@gmail.com; pattap@pchome.com.tw nation showed subscapularis and supraspinatus calcific tendinopathies and supraspinatus tendon partial tear. Due to poor response to physical therapy and intermittent tingling sensation, she was later on referred for an US follow-up. In addition to the previous findings, a well-demarcated anechoic lesion was noted in the spinoglenoid notch adjacent to the suprascapular nerve, thus giving a positive sono-Tinel sign. The spectral Doppler imaging showed a triphasic waveform, compatible with an artery (fig 1A). We did not identify a similar pathology on contra-lateral side examination (fig 1B). It appeared as a pulsatile, tube-like structure under power Doppler imaging (fig 1C). Magnetic resonance imaging (MRI) confirmed the suprascapular nerve entrapment caused by an engorged suprascapular artery (fig 1D) without muscle atrophy.

Suprascapular nerve entrapment is an uncommon cause of chronic shoulder pain that presents with symptoms like numbness, pain and muscle atrophy in longstanding cases. The causes of entrapment include paralabral cysts (being 


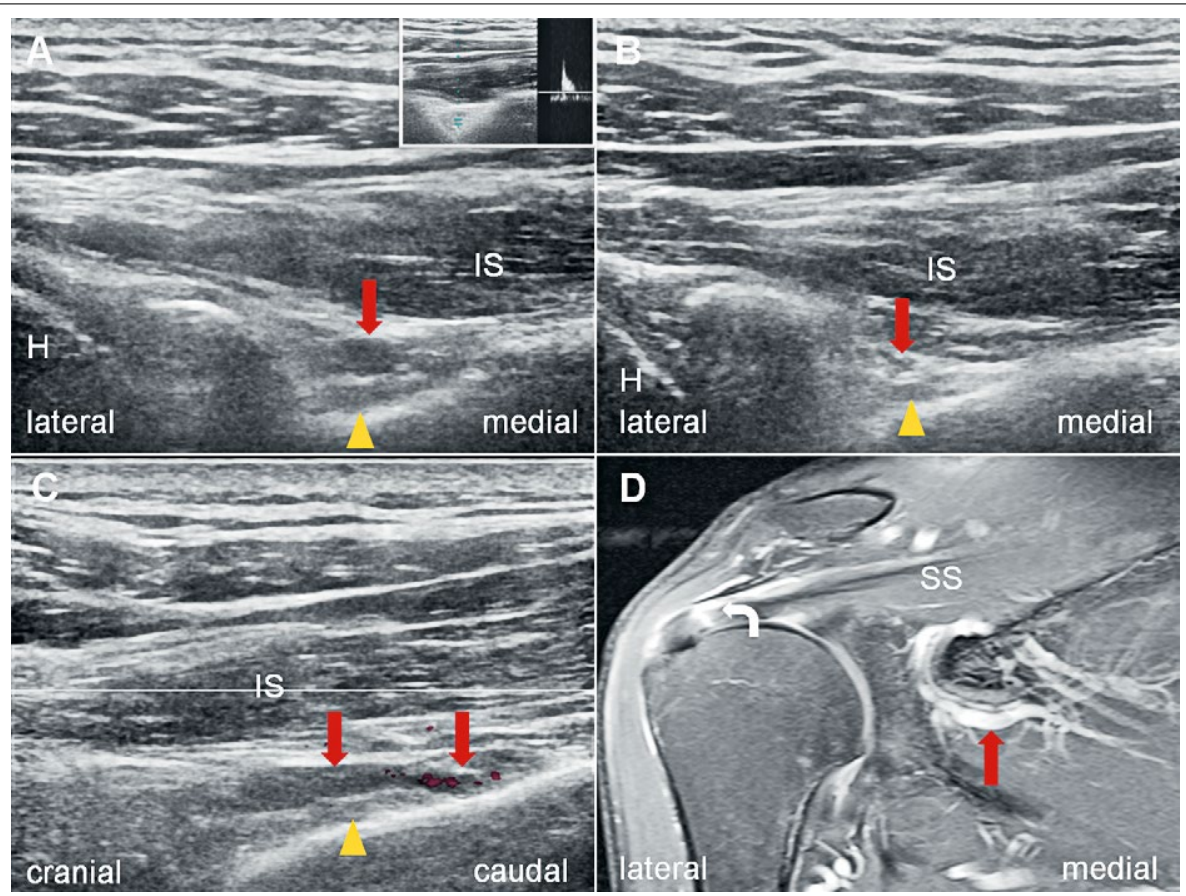

Fig 1. A) Ultrasonographic imaging over the infraglenoid notch (while the probe is being kept parallel to the spine of the scapula) shows the cyst-like lesion (red arrow) next to the suprascapular nerve (yellow arrowhead) at the left shoulder. The spectral Doppler image at right upper corner showing triphasic waveform indicating the suprascapular artery; B) The image at contra-lateral side showing normal finding under short axis view. Red arrow indicating the suprascapular artery; C) Longitudinal view over the lesion showing its curvilinear appearance with some power Doppler signals (red arrows) and the suprascapular nerve (yellow arrowhead); D) Magnetic resonance imaging (sagittal view) showing the suprascapular artery engorgement (red arrow) along the scapular bone. The hyperintense lesion (curved arrow) indicating the supraspinatus tendon tear. H: humeral head; IS: Infraspinatus tendon; SS: supraspinatus tendon.

the most common), vascular abnormalities (usually dilated veins), transverse scapular or spinoglenoid ligament, bony abnormalities and scars of rotator cuff tears [1].

In our case, the underlying etiology causing the compression turned out to be a dilated artery - possibly due to the prior shoulder trauma. While the examiner can evaluate the change of pulsatility and collapse of the suprascapular vein under dynamic US imaging (with internal/ external shoulder rotation) [2], we also want to emphasize the importance of contrast-enhanced MRI because US cannot explore the lesion when the artery travels through the osseous tunnel of the scapular spine. The spectral Doppler imaging is also crucial in differentiating whether the lesion has arterial or venous origin. Concerning treatment, the imaging findings combined with the patient's episodic shoulder pain may need suprascapular nerve release through open or arthroscopic techniques [3].

In short, we emphasize the usefulness of US imaging while investigating the etiology of suprascapular nerve entrapment whereby the precise diagnosis will help and/or guide the physician in choosing the best option for treatment.

Acknowledgments: the present study is supported by National Taiwan University Hospital, Bei-Hu Branch Ministry of Science and Technology (MOST 106-2314-B-002-180-MY3) and Taiwan Society of U1trasound in Medicine.

\section{References}

1. Carroll KW, Helms CA, Otte MT, Moellken S, Fritz R. Enlarged spinoglenoid notch veins causing suprascapular nerve compression. Skeletal Radiol 2003;32:72-77.

2. Park J, Chai JW, Kim DH, Cha SW. Dynamic ultrasonography of the shoulder. Ultrasonography 2017; doi: 10.14366/ usg. 17055 .

3. Lafosse L, Tomasi A, Corbett S, Baier G, Willems K, Gobezie R. Arthroscopic release of suprascapular nerve entrapment at the suprascapular notch: technique and preliminary results. Arthroscopy 2007;23:34-42. 


\section{A new method for ocular ultrasound examination}

\section{Juan de Dios Berná-Serna, Pablo Chico-Sánchez, Juan de Dios Berná-Mestre, Guillermo Carbonell-López del Castillo}

Department of Radiology, Hospital Clínico Universitario Virgen de la Arrixaca, IMIB, Ctra. Madrid-Cartagena, 30120, El Palmar (Murcia), Spain.

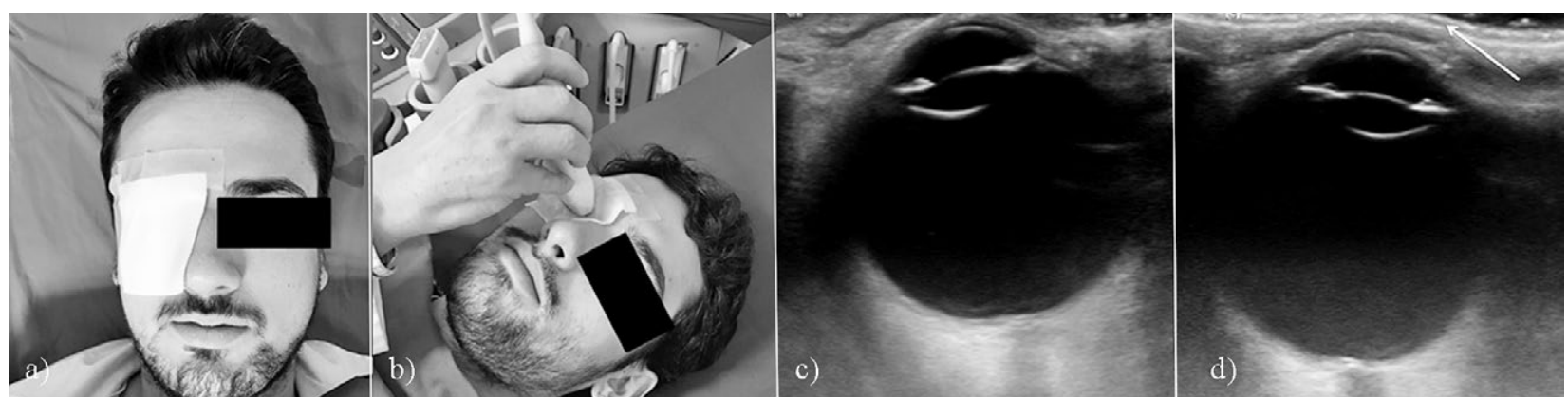

Fig 1. Placement of the moistened gauze over the eye (a). Application of gel on the gauze and positioning the transducer to obtain an axial image of the eye (b). Comparison of images obtained with both methods of ocular B-mode US examination of the same subject. Axial image obtained using standard method (c). Axial image obtained using moistened gauze plus gel method (white arrows in d).

\section{To the Editor}

Ocular B-mode ultrasonography (US) is a widely used technique for assessing a variety of eye conditions, especially when ophthalmoscopy is not possible due to opacification of the transparent [1-4]. US examination is an accessible, easy, rapid, non-invasive and dynamic tool for the evaluation of the eye $[1,4]$.

Usually the first step in ocular B-mode US is to place a generous amount of ultrasound gel on the eyelid to eliminate the air interface. The problem with this procedure, which is performed with the eyes closed and an abundant amount of gel, is the pain or the discomfort reported by patients due to gel seeping into their eyes and to the pressure applied on the eyeball by the examiner with the transducer. Only one study [5] reported the use

Received 07.08.2018 Accepted 12.07.2018

Med Ultrason

2018, Vol. 20, No 3, 402-400, DOI: 10.11152/mu-1640

Corresponding author: Juan de Dios Berná-Serna, MD

Departamento de Radiología, Hospital Clínico

Universitario Virgen de la Arrixaca, Ctra.

Madrid-Cartagena, 30120 El Palmar (Murcia),

Spain

E-mail: jdberna@gmail.com of transparent dressings that allows the gel to be applied on these and not directly on the eyelid, so that the patient's discomfort is potentially eliminated.

The purpose of the present letter to editor is to describe a simple procedure of performing ocular B-mode US using saline solution plus gel moistened gauze.

The ocular US examination consisted in evaluating the eye using the saline-moistened gauze method (GGM) with a 14-5 MHz linear transducer. To perform the eye examination, we previously prepared the saline solution moistened gauze and placed the adhesive plaster shaped in "L". then the gauze was placed over the corresponding eye and secured to the frontal and temporal area with the plaster (fig 1a). Gel was applied on the gauze and the transducer was placed gently on the gel (fig 1b). Finally, cross-sectional and longitudinal images were obtained.

The application of saline-moistened gauze on the eyelid prevents the gel from coming into direct contact with the eye and also prevents the sticky sensation that habitually follows the standard method examination. Furthermore, the GGM obtains images of similar quality to those obtained with the standard method (fig $1 \mathrm{c}, \mathrm{d}$ ). The GGM prevents pain or discomfort during ocular B-mode 
US examination. Moreover, the procedure is simple, safe and well tolerated.

\section{References}

1. Bedi DG, Gombos DS, Ng CS, Singh S. Sonography of the eye. AJR Am J Roentgenol 2006;187:1061-1072.

2. Kilker BA, Holst JM, Hoffmann B. Bedside ocular ultrasound in the emergency department. Eur J Emerg Med 2014;21:246-253.
3. De La Hoz Polo M, Torramilans Lluís A, Pozuelo Segura O, Anguera Bosque A, Esmerado Appiani C, Caminal Mitjana JM. Ocular ultrasonography focused on the posterior eye segment: what radiologists should know. Insights Imaging 2016;7:351-364.

4. Dudea SM. Ultrasonography of the eye and orbit. Med Ultrason 2011;13:171-174.

5. Roth KR, Gafni-Pappas G. Unique method of ocular ultrasound using transparent dressings. J Emerg Med 2011;40:658-660.

\title{
Ultrasound imaging for lateral knee pain: popliteus tendon highlighted
}

\author{
Vincenzo Ricci' ${ }^{1}$, Levent Özçakar ${ }^{2}$
}

${ }^{1}$ IRCCS Rizzoli Orthopaedic Institute, Department of Biomedical and Neuromotor Science, Physical and Rehabilitation Medicine Unit, Bologna, Italy, ${ }^{2}$ Hacettepe University Medical School, Department of Physical and Rehabilitation Medicine, Ankara, Turkey

\section{To the Editor,}

A 45-year-old male patient was evaluated due to right knee pain for the past four weeks. The pain worsened during running activities especially in the postero-lateral side of the joint, referring to it as a "feeling of constriction" around the knee. He denied any trauma or episodes of joint locking and declared that nonsteroidal anti-inflammatory drugs had been partially effective. Physical examination revealed pain in the popliteal cord and on the lateral side of the knee with negative McMurry and Apley tests. Ultrasound (US) imaging was also performed in accordance with the EURO-MUSCULUS/USPRM knee scanning protocol [1]. In addition to the slight amount of fluid in the suprapatellar recess and synovial hypertrophy of the lateral para-meniscal recesses, a remarkable fluid distension surrounding the popliteus tendon (PT) in the groove of the lateral femoral condyle were observed (fig 1). An oral nonsteroidal anti-inflammatory drug and

Received 12.06.2018 Accepted 10.07.2018

Med Ultrason

2018, Vol. 20, No 3, 403-400, DOI: 10.11152/mu-1616

Corresponding author: Vincenzo Ricci

IRCCS Rizzoli Orthopaedic Institute,

Department of Biomedical and Neuromotor

Science, Physical and Rehabilitation Medicine

Unit, Bologna, Italy

E-mail: vincenzo.ricci58@gmail.com low-level laser-therapy were prescribed for 1 week, followed by a personalized rehabilitation program with specific stretching exercises for the popliteus muscle and the iliotibial band (ITB). After 3 weeks, the patient reported significant pain relief and considerable improvement in walking and running.

Lateral knee pain is really common, especially in runners, whereby the differential diagnosis includes ITB syndrome, lateral meniscal pathology, patella-femoral syndrome, biceps femoris and popliteus tendinopathies [2]. Biomechanically, PT is a major stabilizer of the posterolateral knee and overtraining can lead to extra friction between the popliteus tendon, lateral femoral condyle and the lateral meniscus, eventually causing inflammation of the superior lateral para-meniscal recess, which normally functions as an "incomplete sheath" for the PT. Anatomical studies have shown that the popliteal recess is an extra-articular extension of the synovial membrane of the knee joint and that this synovial recess extends from the popliteal hiatus along the proximal part of the PT [3].

Keeping in mind the fact that the capsule-synovial complex is a strongly innervated structure, the synovial popliteus recess is a critical potential pain generator that should be scanned diligently during the assessment of postero-lateral painful knee. Herein, we aimed to underscore that - although there is wide use of US for imaging and guided interventions of knee joint - some structures 


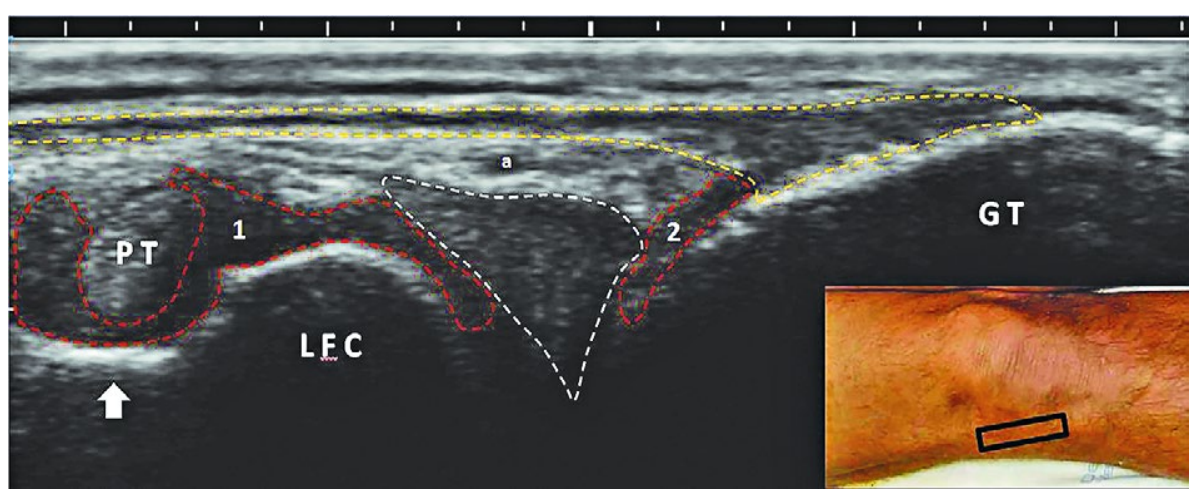

Fig 1. Ultrasound imaging shows the iliotibial band (dotted yellow line), lateral meniscus (dotted white line), popliteus tendon (PT) and its groove on the lateral femoral condyle (white arrow). Superior (1) and inferior (2) para-meniscal recesses are marked with dotted red lines. Inset shows the position of the probe during scanning of the lateral knee in coronal oblique view. LFC: lateral femoral condyle, GT: Gerdy’s tubercle, a: lateral inferior genicular artery

are not routinely scanned in the daily US knee examinations and also that they might actually be the culprits of recalcitrant knee pain [4].

\section{References}

1. Özçakar L, Kara M, Chang KV, et al. EURO-MUSCULUS /USPRM Basic scanning protocols for knee. Eur J Phys Rehabil Med 2015;51:641-646.
2. Smith J, Finnoff JT, Sante BS, et al. Sonographically guided popliteus tendon sheat injection. J Ultrasound Med 2010;29:775-782.

3. Jadhav SP, More SR, Riascos RF, et al. Comprehensive review of the anatomy, function, and imaging of the popliteus and associated pathologic conditions. Radiographics 2014;34:496-513.

4. Chang KV, Wu WT, Özçakar L. Ultrasound-guided diagnosis and intervention for painful knee: sonoanatomy revisited. Pain Manag doi: 10.2217/pmt-2018-0014. 